\title{
In Vivo MRI of Endogenous Remyelination in a Nonhuman Primate Model of Multiple Sclerosis
}

4 Nathanael J. Lee, ${ }^{1,2}$ Pascal Sati, ${ }^{1,3}$ Seung-Kwon Ha, ${ }^{1,4}$ Nicholas J. Luciano, ${ }^{1}$ Snehashis Roy, ${ }^{5}$

5 Benjamin Ineichen, ${ }^{1,6}$ Emily C. Leibovitch, ${ }^{7}$ Cecil C. Yen, ${ }^{8}$ Dzung L. Pham, ${ }^{9}$ Afonso C. Silva, ${ }^{4,8}$

6 Mac Johnson, ${ }^{10}$ Steven Jacobson, ${ }^{7}$ Daniel S. Reich ${ }^{1} * *$

$7 \quad{ }^{1}$ Translational Neuroradiology Section, ${ }^{7}$ Viral Immunology Section, and ${ }^{8}$ Cerebral Microcircula8 tion Section, Laboratory of Functional and Molecular Imaging, National Institute of Neurologi-

9 cal Disorders and Stroke, National Institutes of Health, Bethesda, MD, USA

$10{ }^{2}$ Department of Medicine, Massachusetts General Hospital / Harvard Medical School, Boston, 11 MA USA

$12{ }^{3}$ Neuroimaging Program, Department of Neurology, Cedars Sinai, Los Angeles, CA, USA

$13{ }^{4}$ Department of Neurobiology, University of Pittsburgh, Pittsburgh, PA, USA

$14{ }^{5}$ Section on Neural Function, National Institute of Mental Health, National Institutes of Health, 15 Bethesda, MD, USA

16
${ }^{6}$ Department of Neuroradiology, Clinical Neuroscience Center, University Hospital Zurich, University of Zurich, Switzerland

${ }^{9}$ Center for Neuroscience and Regenerative Medicine, The Henry M. Jackson Foundation for the Advancement of Military Medicine, Bethesda, MD, USA

${ }^{10}$ Vertex Pharmaceuticals Incorporated, Boston, MA USA

*Equal contribution

**Corresponding author

- Email: daniel.reich@ nih.gov

- Mailing address: Building 10, Room 5C103, 10 Center Drive MSC 1400, Bethesda, Maryland, USA 20892

- Telephone: +1-301-496-1801

- Fax: +1-301-402-0373 
bioRxiv preprint doi: https://doi.org/10.1101/2021.10.27.466044; this version posted October 28, 2021. The copyright holder for this preprint (which was not certified by peer review) is the author/funder, who has granted bioRxiv a license to display the preprint in perpetuity. It is made available under aCC-BY 4.0 International license.

Page 2 of 31 


\section{ABSTRACT}

33 Remyelination is crucial for recovery from inflammatory demyelination in multiple sclerosis

34 (MS). Investigating remyelination in vivo using magnetic resonance imaging (MRI) is difficult in

35 MS, where collecting serial short-interval scans is challenging. Using experimental autoimmune

36 encephalomyelitis (EAE) in common marmosets, a model of MS that recapitulates focal cerebral

37 MS lesions, we investigated whether remyelination can be detected and characterized noninva-

38 sively. In 6 animals followed with multisequence 7-tesla MRI, 36 focal lesions, classified as de-

39 myelinated or remyelinated based on signal intensity on proton density-weighted images, were

40 subsequently assessed with histopathology. Remyelination occurred in 5 of 6 marmosets and

$4151 \%$ of lesions. Radiological-pathological comparison showed high sensitivity (88\%) and speci-

42 ficity (90\%) for detecting remyelination by in vivo MRI. This study demonstrates the prevalence

43 of spontaneous remyelination in marmoset EAE and the ability of in vivo MRI to detect it, with

44 implications for preclinical testing of pro-remyelinating agents and translation to clinical prac45 tice. 


\section{INTRODUCTION}

50 Multiple sclerosis (MS) is a debilitating inflammatory demyelinating disorder affecting millions

51 worldwide (1). MS causes dynamic changes to myelin in the central nervous system (CNS), in-

52 cluding the quintessential focal inflammatory destruction of myelin, as well as the phenomenon

53 of remyelination that can follow the demyelination (2-5). Although remyelination is a crucial

54 aspect of tissue repair, and as such represents an important therapeutic target (6), most

55 knowledge about remyelination in MS derives from postmortem studies using histochemical and

56 electron microscopy studies. This is because investigating remyelination in vivo in real time is

57 limited by imperfect discrimination on neuroimaging modalities such as magnetic resonance

58 imaging (MRI). Furthermore, in human beings, where collecting serial short-interval scans is

59 highly challenging, it is difficult to detect track the dynamic occurrence of remyelination. There-

60 fore, to investigate the pathobiology of remyelination in the context of focal inflammatory demy-

61 elination, a reliable preclinical model is needed to develop techniques that can then be applied

62 clinically.

63

64 Rodent models have been widely used to investigate various aspects of the pathobiology of de-

65 myelination. However, while toxin models in mice, including the lysolecithin and cuprizone

66 models, display demyelination and even remyelination, they do not require involvement of adap-

67 tive immune cells $(7,8)$. Conversely, rodent experimental autoimmune encephalomyelitis (EAE)

68 models, while mediated by an immune response, are often neither focal nor profoundly demye-

69 linating. There is no known rodent model that is characterized by multifocal inflammatory de-

70 myelination in the brain that is disseminated in both space and time. 
72 EAE in the common marmoset (Callithrix jacchus) is a well-recognized translational model that

73 serves as a bridge between the rodent EAE and human MS (9-11). Not only does EAE recapitu-

74 late MS at the lesion level both radiologically and pathologically $(12,13)$, but lesions spontane-

75 ously remyelinate (14), as occurs in MS.

76

77 Prior studies demonstrated that certain signal changes in MRI, such as magnetization transfer

78 ratio (MTR), correlate with remyelination in MS lesions (15-19). This has also been investigated

79 in animal models, albeit mainly in the rodent model and in the cortex $(20,21)$ rather than white

80 matter. It has also been demonstrated that partial remyelination can occur and can be localized

81 either to specific parts of the lesion (most commonly the lesion edge) or to the whole lesion (22,

82 23).

83

84 Here, we studied focal white matter lesions in marmoset EAE. We utilized serial in vivo MRI, 85 mainly involving proton density-weighted (PDw) and MTR sequences, to age and characterize 86 lesions. We further analyzed the lesions using histopathology, focusing on myelin lipids and pro87 teins, to compare and study the reliability of using various in vivo MRI sequences in predicting remyelination and their histopathological correlates. 


\section{METHODS}

\section{Marmoset EAE induction}

926 marmosets (3 pairs of twins; 4 males and 2 females, ages $2-6$ at baseline) were included in the

93 study (Table 1). 2 marmosets (M\#1-2) first received 100-mg of human white matter homogenate

94 followed by an additional $200 \mathrm{mg}$ after no lesions were detected by in vivo MRI 2 months after

95 the initial injection $(14,24) .4$ additional marmosets (M\#3-6) received $200 \mathrm{mg}$ of white matter

96 homogenate, specifically from the temporal lobe, collected from autopsy. All white matter ho-

97 mogenates were mixed with complete Freund's adjuvant (Difco Laboratories). Prior marmoset

98 EAE studies have merged data from different induction protocols in an effort to make the most

99 of this rare animal resource, and there is no evidence that lesion-level pathology differs between

100 the two protocols used here, or between the sexes (25). In each twin pair, the first animal to de-

101 velop a lesion, detected by in vivo MRI, received intravenous methylprednisolone (18 mg/kg/day

102 for 5 consecutive days) with the goal to reduce the severity of inflammation, potentially allowing

103 longer-term evaluation of the lesions; this regimen is based on treatment of acute MS relapses

$104(26,27)$. Experiments were terminated when animals became either paraplegic or lost more than

$10520 \%$ of their baseline body weight. For psychosocial wellbeing, all marmosets were housed with

106 another marmoset (usually a twin counterpart). Animals were weighed and monitored on a daily

107 basis to ensure adequate nutritional intake and physical wellbeing. All protocols were approved

108 by the National Institutes of Neurological Disorders and Stroke Animal Care and Use Commit-

109 tee. 


\begin{tabular}{ccccccc}
\hline \hline Animal & Sex & $\begin{array}{c}\text { Age } \\
\text { (years) }\end{array}$ & $\begin{array}{c}\text { 1st immun- } \\
\text { ization }\end{array}$ & $\begin{array}{c}\mathbf{2}^{\text {nd }} \text { immun- } \\
\text { ization }\end{array}$ & $\begin{array}{c}\text { Corticosteroid } \\
\text { treatment }\end{array}$ & $\begin{array}{c}\text { Experiment } \\
\text { duration } \\
\text { (weeks) }\end{array}$ \\
\hline M\#1* $^{*}$ & Male & 3.7 & $100 \mathrm{mg}$ & $200 \mathrm{mg}$ & Yes & 40 \\
\hline $\mathbf{M \# 2}^{*}$ & Male & 3.7 & $100 \mathrm{mg}$ & $200 \mathrm{mg}$ & No & 54 \\
\hline M\#3 $^{+}$ & Male & 6.2 & $200 \mathrm{mg}$ & - & Yes & 61 \\
\hline $\mathbf{M \# 4}^{+}$ & Male & 6.2 & $200 \mathrm{mg}$ & - & No & 60 \\
\hline M\#5 $^{+}$ & Female & 2.8 & $200 \mathrm{mg}$ & - & Yes & 18 \\
\hline M\#6 $^{\#}$ & Female & 2.8 & $200 \mathrm{mg}$ & - & No & 16 \\
\hline \hline
\end{tabular}
Table 1. Demographics and experimental information for the 6 marmosets included in this study. Immunizations used human white matter homogenate. Experiment duration corresponds to the time between immunization and terminal MRI. * ${ }^{+},{ }^{\mathbb{H}}$ denote the 3 different pairs of twin animals.

\section{Marmoset in vivo MRI}

All 6 marmosets were scanned on a weekly basis under anesthesia, as previously described (12, 14, 24). We used PDw contrast, which is sensitive to demyelination (28), to visualize lesions in vivo. T1-weighted $(\mathrm{T} 1 \mathrm{w})$, T2-weighted $(\mathrm{T} 2 \mathrm{w}), \mathrm{T} 2 *^{-}$-weighted $\left(\mathrm{T} 2 *_{\mathrm{w}}\right)$ and MTR contrasts were also obtained (Figure 1). T1w contrast was repeated after injection of gadolinium-based contrast agent (gadobutrol, $0.3 \mathrm{mg} / \mathrm{kg}$ ) to visualize enhancing lesions. Specific sequence parameters for the different MRI contrasts are listed on Table 2. Images were post-processed using an in-house pipeline, including intensity correction, image cropping, multi-contrast registration, skullstripping, and intensity normalization (Supplementary Figure 1). To minimize harm and pain in animals, all procedures, including intravenous access for gadolinium injection, were done under anesthesia. For post-anesthesia recovery, animals were gently woken up using warm blankets, and returned to their respective housing only after the animals were back to pre-anesthesia baseline, including spontaneous breathing, physical activities, and intractability. 


\begin{tabular}{|c|c|c|c|c|c|}
\hline MRI contrast & PDw & T1w & T2w & $\mathbf{T} 2 * \mathbf{w}$ & $\overline{\text { MTR }}^{+}$ \\
\hline Sequence & 2D RARE & 2D MDEFT & 2D RARE & 2D MGE & 3D MGE \\
\hline FOV (mm) & $32 \times 24$ & $32 \times 24$ & $32 \times 24$ & $32 \times 24$ & $38.4 \times 38.4 \times 36$ \\
\hline Matrix & $214 \times 160$ & $214 \times 160$ & $214 \times 160$ & $214 \times 160$ & $256 \times 256 \times 36$ \\
\hline Number of slices & 36 & 36 & 36 & 36 & 36 \\
\hline $\begin{array}{l}\text { Slice thickness } \\
(\mathrm{mm})\end{array}$ & 1 & 1 & 1 & 1 & 1 \\
\hline TR (ms) & 2300 & 12.5 & 8000 & 2150 & 20 \\
\hline $\mathrm{TE}(\mathrm{ms})$ & 16 & 4.2 & 72 & 18 & 5 \\
\hline $\mathrm{TI}(\mathrm{ms})$ & N/A & 1200 & N/A & N/A & N/A \\
\hline ETL & 1 & N/A & 4 & N/A & N/A \\
\hline $\begin{array}{l}\text { Exc. Pul. (shape, } \\
\text { FA) }\end{array}$ & Sinc $3,90^{\circ}$ & Sinc $3,12^{\circ}$ & Sinc $3,90^{\circ}$ & Sinc $3,70^{\circ}$ & Sinc $3,10^{\circ}$ \\
\hline $\begin{array}{l}\text { Ref. Pul. (shape, } \\
\text { FA) }\end{array}$ & Sinc $3,180^{\circ}$ & N/A & Sinc $3,180^{\circ}$ & N/A & N/A \\
\hline $\begin{array}{c}\text { Pre. Pul. (type, } \\
\text { shape, FA, offset) }\end{array}$ & N/A & $\begin{array}{l}\text { Exc./Inv., Sech, } \\
90^{\circ} / 180^{\circ}\end{array}$ & N/A & N/A & $\begin{array}{l}\text { MT, Gauss, } \\
1500 \mathrm{~Hz}\end{array}$ \\
\hline NEX & 1 & 1 & 2 & 1 & 2 \\
\hline AT & $\begin{array}{c}7 \min 40 \\
\sec \end{array}$ & $6 \mathrm{~min} 56 \mathrm{sec}$ & $\begin{array}{c}13 \min 20 \\
\sec \end{array}$ & $7 \mathrm{~min} 10 \mathrm{sec}$ & $7 \mathrm{~min} 58 \mathrm{sec}$ \\
\hline
\end{tabular}

Table 2. Main parameters used for the different MRI contrasts acquired in vivo. FOV = field of view; $\mathrm{TR}=$ repetition tine; $\mathrm{TE}=$ echo time; $\mathrm{TI}=$ inversion time; $\mathrm{ETL}=$ echo train length (or RARE factor); Exc. Pul. = excitation pulse; Ref. Pul. = refocusing pulse; Pre. Pul. = preparation pulse, FA $=$ flip angle; NEX $=$ number of repetitions; $\mathrm{AT}=$ acquisition time; $\mathrm{RARE}=$ rapid acquisition with relaxation and enhancement; MDEFT $=$ modified drive equilibrium Fourier transform; MGE $=$ multi gradient echo.

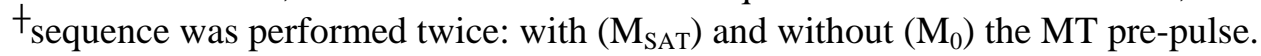




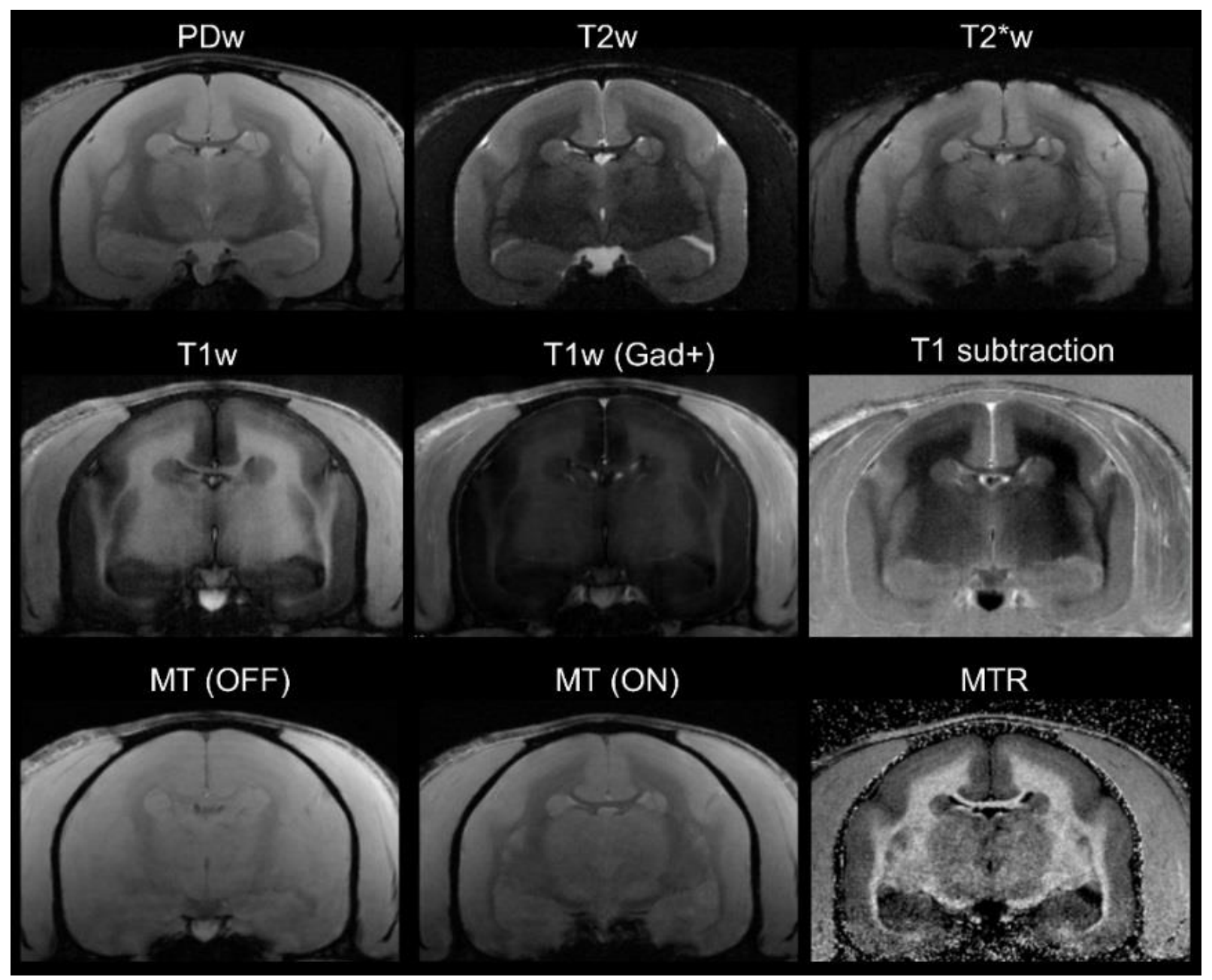

Figure 1. In vivo (baseline) multicontrast marmoset brain MRI. T1 subtraction image was obtained from subtracting pre-gadolinium T1w from post-gadolinium T1w images. MTR image derived voxelwise as $\left(M_{0}-M_{S A T}\right) / M_{0}$. Animal M\#5.

\section{Histopathology of EAE lesions}

Marmoset brains were collected immediately after death once the animals met the study endpoint. Brain tissue was processed using formalin-fixation and paraffin-embedding and subsequent histopathological staining, as described previously $(14,24)$. Postmortem histological processing failed for animal M\#1's brain. For visualizing myelin, Luxol fast blue (LFB) staining with periodic acid Schiff (PAS) counterstain and immunohistochemistry for myelin proteolipid protein (PLP) were used. For characterizing inflammation, edema, gliotic activity, hematoxylin 
148

149

150

151

152

153

154

155

156

157

158

159

160

161

162

163

164

165

166

167

168

169

170

and eosin $(\mathrm{H} \& \mathrm{E})$ and immunohistochemistry for ionized calcium-binding adaptor molecule (Iba1) were used. Oligodendrocytes and oligodendrocyte precursor cells (OPC) were assessed with aspartoacylase (ASPA) and oligodendrocyte transcription factor (Olig2) double staining (mature oligodendrocytes were ASPA and Olig2 positive; OPCs were Olig2 positive but ASPA negative). For axon staining, Bielschowsky's silver method was used. Briefly, deparaffinized slides were covered with $20 \% \mathrm{AgNO}_{3}$ and incubated at $40{ }^{\circ} \mathrm{C}$ inside a dark chamber for 30 minutes. Slides were washed and placed in ammonia silver solution, prepared by adding concentrated ammonium hydroxide drop-by-drop into $\mathrm{AgNO}_{3}$ until brown precipitate disappeared, at 40 ${ }^{\circ} \mathrm{C}$ for 30 minutes. Developer working solution was added to the slides, made with developer stock solution (37-40\% formaldehyde, citric acid, and nitric acid), ammonium hydroxide, and distilled water. After all incubations, slides were washed with $1 \%$ ammonium hydroxide, washed in distilled water, and treated with 5\% sodium thiosulfate solution. Detailed immunohistochemical information is provided in Supplementary Table 1.

\section{In vivo MRI analysis of EAE lesions}

Focal marmoset EAE lesions in the white matter were detected on PDw images using an automated convolutional neural network $(\mathrm{CNN})$-based segmentation algorithm $(29,30)$. The network was trained using PDw MRI images from 3 EAE marmosets along with binary manual segmentation of lesions. An atlas consists of a pair of postprocessed PDw images (baseline and a timepoint) and the binary lesion segmentation of that time-point. The baseline is assumed to be lesion free. Once the network was trained, it was applied to the serial PDw images collected on our 6 animals (Supplementary Figure 2), and lesion masks were automatically generated. Any lesion smaller than 4 voxels $(0.0225 \mu \mathrm{l})$ was removed from analysis. For temporal progression compu- 
171 tation, a lesion at timepoint $t=t_{1}$ was identified as the same lesion at timepoint $t=t_{2}$ if they over-

172 lapped by at least 4 voxels in 3D. All automated lesion segmentations were reviewed by an expe-

173 rienced rater. For subsequent MRI analysis of lesion trajectories, including intensity changes

174 over time, all lesions were identified on every scan of each marmoset, and the average intensities

175 were calculated using the automatically segmented lesions. For pre-lesion timepoints, a region of

176 interest (ROI) was drawn manually and positioned in the normal appearing white matter area

177 where the lesion later appeared.

\section{EAE lesion characterization, categorization, and comparison}

181 EAE lesions were characterized independently and separately based on in vivo MRI characteris182 tics and histopathological analyses (2 separate experienced raters for MRI, and 2 experienced raters for histopathological analysis, blinded to each other). The lesions were categorized as follows: acute demyelinating, chronic demyelinated, and remyelinated. Once the lesions were independently analyzed, the results were directly compared for statistical analyses as described be-

186 low.

\section{Statistical analysis}

189 To evaluate the sensitivity and specificity of in vivo MRI detection of demyelination or remye-

190 lination, relative to by histopathology, we created confusion matrices and calculated true or false 191 positive and negative rates. For interrater reliability of using MRI to predict remyelination, Co-

192 hen's kappa was calculated. To test the effects of corticosteroid treatment and sex on remye193 lination, the point biserial correlation model was used. 
bioRxiv preprint doi: https://doi.org/10.1101/2021.10.27.466044; this version posted October 28, 2021. The copyright holder for this preprint (which was not certified by peer review) is the author/funder, who has granted bioRxiv a license to display the preprint in perpetuity. It is made available under aCC-BY 4.0 International license.

195

Page 12 of 31 


\section{RESULTS}

\section{Lesion characterization and categorization}

Typical acute demyelinating lesions were hyperintense on PDw images until the terminal scan,

grew rapidly to several cubie millimeters immediately after establishment, and then showed min-

A.

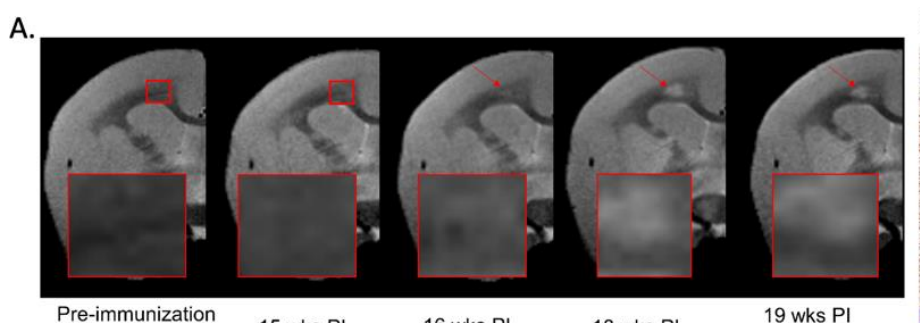

B.
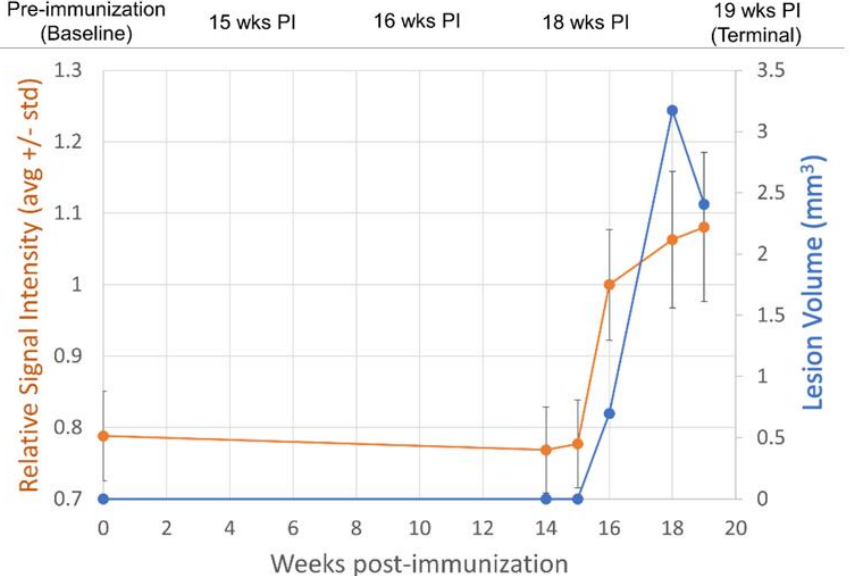

c.

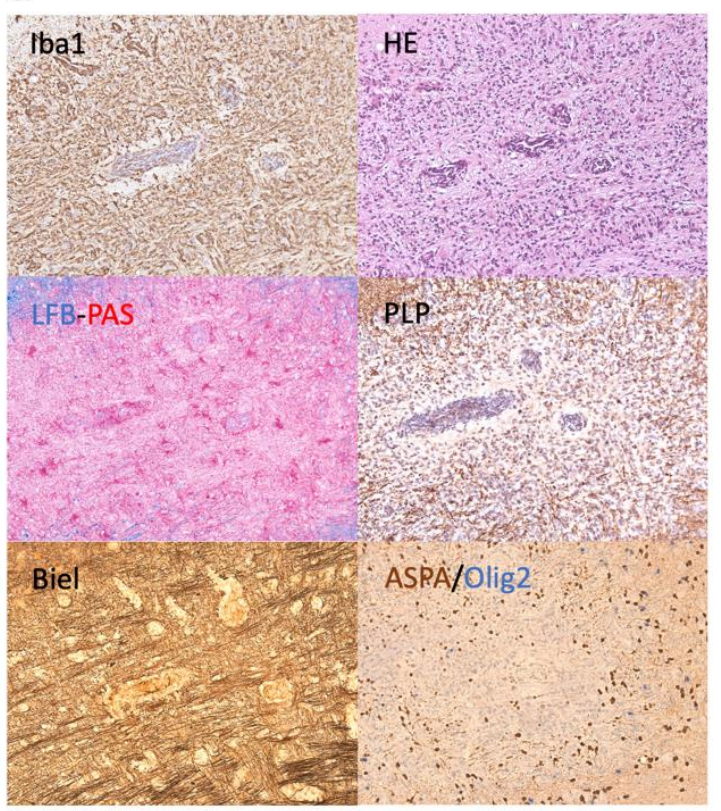

Figure 2. Acute demyelinating EAE lesion with persistent hyperintensity on PDw MRI and active inflammatory demyelination on histopathology. (A) In vivo MRI of proton density-weighted (PDw) acquired before EAE induction (baseline) and at various timepoints leading up to terminal scan. Images were processed as described in Methods. Red arrows = focal white matter lesion first detected 16 weeks after immunization, which persisted through the terminal MRI. Red boxes = magnified insets. (B) Temporal evolution of volume (blue line) and PDw signal (orange line) of the segmented lesion. Lesion signal intensity is normalized to gray matter signal intensity, which was set to 1. (C) Histochemical panel at 
bioRxiv preprint doi: https://doi.org/10.1101/2021.10.27.466044; this version posted October 28, 2021. The copyright holder for this preprint (which was not certified by peer review) is the author/funder, who has granted bioRxiv a license to display the preprint in perpetuity. It is made available under aCC-BY 4.0 International license.

200X magnification of the same lesion, demonstrating active inflammatory demyelination and edema, loss of oligodendrocytes, and axon loss. Hematoxylin counterstaining used for PLP and Iba1. Lesion selected from M\#6.

\section{Typical chronic demyelinated lesions demonstrated long-lasting hyperintensity on PDw images}

until the terminal scan with resolution of gadolinium enhancement on T1w images (Figure 3A-

B). PLP staining showed sharp lesion borders with complete demyelination on LFB-PAS (Figure

3C). Lesions contained clusters of $\mathrm{Iba}^{+}$cells. ASPA/Olig2 staining revealed loss of both mature

oligodendrocytes and OPC. H\&E staining showed less edema compared to acute demyelinating

lesions, and Bielschowsky silver stain demonstrated loss of axons.

A.

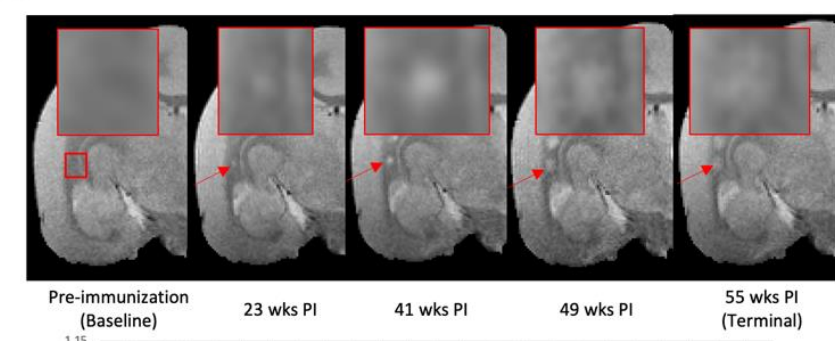

B.

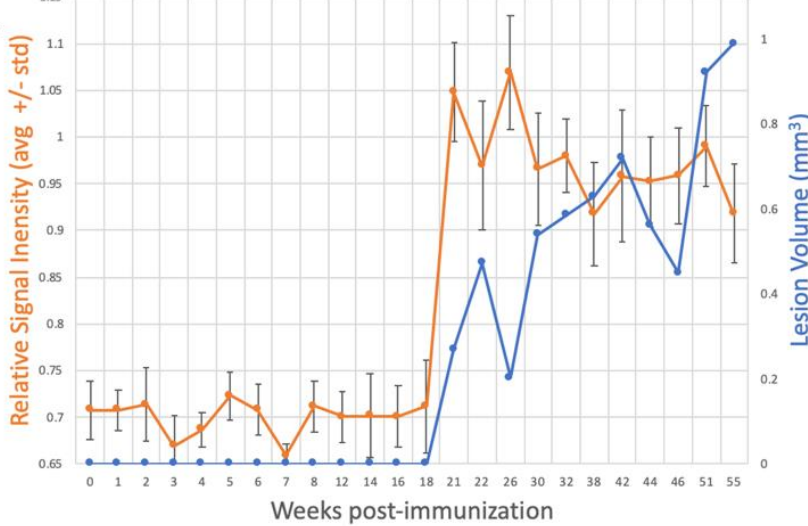

C.

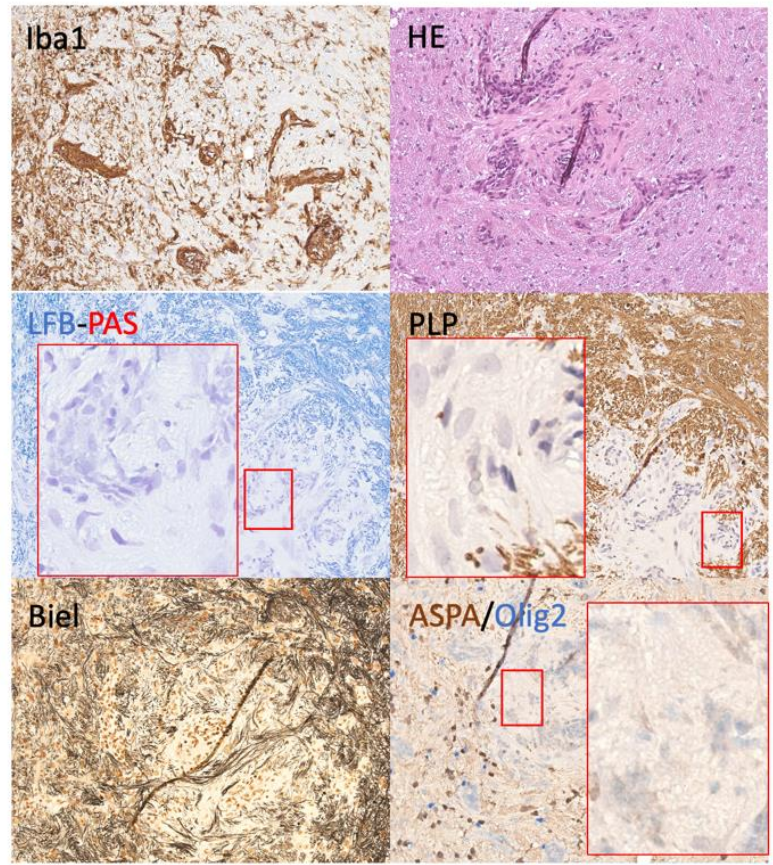

Figure 3. Chronic demyelinated EAE lesion with long-lasting hyperintensity on serial PDw MRI and complete loss of myelin on histopathology. (A) In vivo MRI of proton density-weighted (PDw) acquired before EAE induction (baseline) and at various timepoints leading up to terminal scan. Images were processed as described in Methods. Red arrows $=$ focal white matter lesion first detected 23 weeks after immunization, which persisted until the terminal MRI. Red boxes = magnified insets. (B) Temporal evolution of volume (blue line) and PDw signal (orange line) of the segmented lesion, with acute and persistent increase in signal intensity and increasing lesion volume. Lesion signal intensity is normalized relative to gray matter signal intensity, which was set to 1. (C) Histochemical panel at 200X magnification of the same lesion showing loss of myelin, axons, and oligodendrocytes, as well as persistent inflammation. Red boxes = magnified insets. Hematoxylin counterstaining used for PLP and Iba1. Lesion selected from M\#3. 
A.

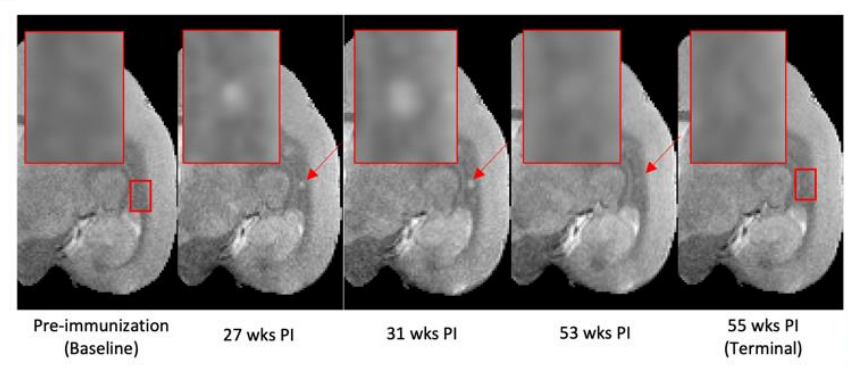

B.

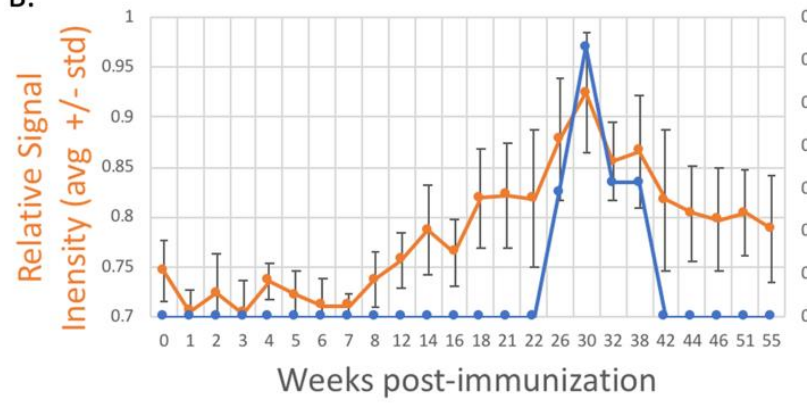

C.

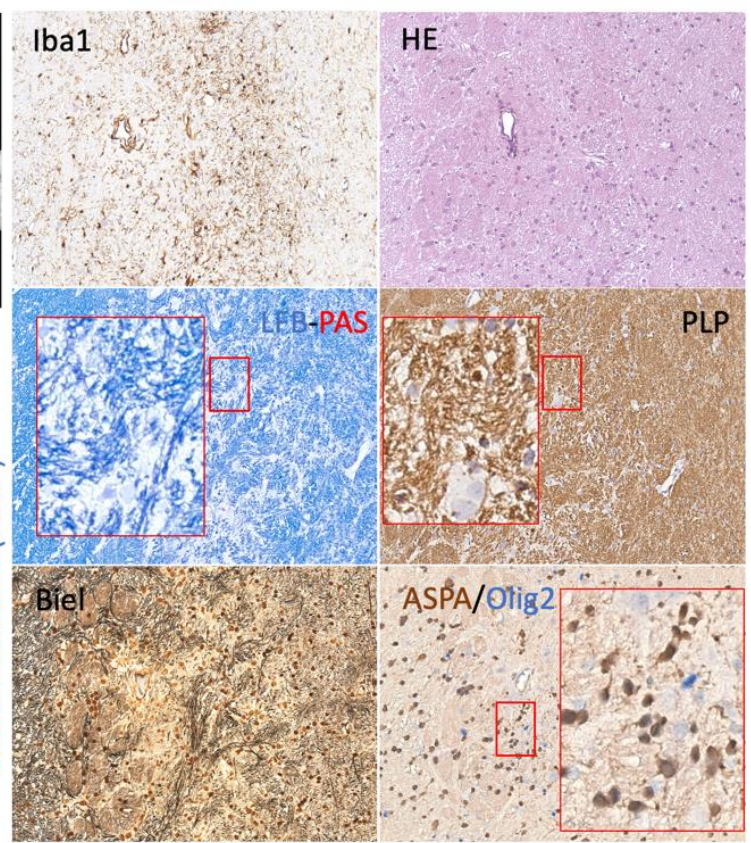

Figure 4. Remyelinated EAE lesion with initial hyperintensity that returned to isointensity. (A) In vivo proton density-weighted (PDw) MRI acquired before EAE induction (baseline) and at various timepoints leading up to the terminal scan. Images were processed as described in Methods. Red arrows = focal white matter lesion first detected 27 weeks post immunization (PI), which appeared to subsequently resolve on MRI. Red boxes = magnified insets. (B) Temporal evolution of volume (blue line) and PDw signal (orange line) of the segmented lesion. Signal intensity peaked around 31 weeks PI and returned to baseline by 42 weeks PI. Lesion signal intensity is normalized relative to gray matter signal intensity, which was set to 1. (C) Histochemical panel at 200X magnification of the same lesion showing sparse myelin and presence of both oligodendrocytes on OPC, as well as less inflammation and edema than other lesion types. Axons appeared closer to within normal limits compared to demyelinated lesions. Red boxes = magnified insets. Hematoxylin counterstaining used for PLP and Iba1. Lesion selected from M\#3. 


\section{Spontaneous remyelination is common in marmoset EAE}

Using in vivo MRI only, 43 focal white matter lesions were detected in the 6 EAE marmosets

Based on the histopathological classification criteria, 36 lesions were identified in the 5 animals demyelinating (25\%), and 10 as chronic demyelinated (28\%). All 3 animals with putative remye-

277 single remyelinated lesion not seen on MRI; M\#6 only demonstrated acute and chronic demye-

278 linated lesions, consistent with the MRI.

\begin{tabular}{ccccc}
\hline \hline Animal & $\begin{array}{c}\text { Acute Demye- } \\
\text { linating }\end{array}$ & $\begin{array}{c}\text { Chronic De- } \\
\text { myelinated }\end{array}$ & Remyelinated & Total \\
\hline M\#2 & 0 & 1 & 9 & 10 \\
\hline M\#3 & 0 & 1 & 5 & 6 \\
\hline M\#4 & 0 & 1 & 2 & 3 \\
\hline M\#5 & 4 & 4 & 1 & 9 \\
\hline \hline
\end{tabular}




\begin{tabular}{ccccc}
\hline \hline M\#6 & 5 & 3 & 0 & 8 \\
\hline Total & 9 & 10 & 17 & 36 \\
\hline \hline
\end{tabular}

Table 4: Classification of focal lesions by histopathology.

\section{Remyelination is reliably detected by in vivo MRI}

Of the 36 focal white matter lesions identified on both in vivo PDw MRI and histopathology, classification was concordant for 26 lesions (72\%). Of these, 5 (19\%) were acute demyelinating, $6(23 \%)$ were chronically demyelinated, and 15 were remyelinated $(58 \%)$. When the lesions were grouped by myelination status only (remyelinated vs. demyelinated), in vivo PDw MRI correctly predicted 32 out of 35 lesions, showing 89\% agreement (Table 5). Using histopathology as the gold standard, there was $88 \%$ sensitivity and $90 \%$ specificity for detecting remyelination. Interrater reliability for use of serial PDw MRI to detect remyelination was 94\%, with

\begin{tabular}{|c|c|c|c|}
\hline$n=36$ & $\begin{array}{c}\text { Predicted demye- } \\
\text { lination by MRI }\end{array}$ & $\begin{array}{c}\text { Predicted remyelination by } \\
\text { MRI }\end{array}$ & \\
\hline $\begin{array}{c}\text { Actual demyelination } \\
\text { by histopathology }\end{array}$ & 17 & 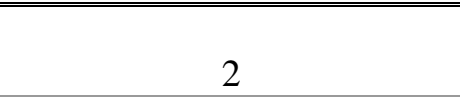 & 19 \\
\hline $\begin{array}{c}\text { Actual remyelination } \\
\text { by histopathology }\end{array}$ & 2 & 15 & 17 \\
\hline & 19 & 17 & \\
\hline
\end{tabular}

Table 5: Confusion matrix for PDw MRI classification of demyelinated vs. remyelinated lesions 298 compared to histopathology.

299 MTR was separately used to classify lesions, in order to compare its utility to predict myelin status with PDw. The sensitivity and specificity for detecting remyelination, using histopathology as the standard, were $82 \%$ and $79 \%$, respectively, both lower than for PDw. 


\section{Remyelination is a time-specific event in marmoset EAE}

304 Based on our frequent longitudinal MRI-based sampling and aging of EAE lesions and analysis

305 of signal intensity, with comparison to histopathology, we found that inflammation and demye-

306 lination are the primary components of lesion pathophysiology in lesions $<5$ weeks old, corrobo-

307 rating our previous work $(14,24)$. The earliest remyelination detected was 5 weeks after initial

308 lesion detection on in vivo MRI, although most remyelinated lesions were $>7$ weeks old. To es-

309 timate the rate of remyelination, we performed an initial estimate in 4 focal lesions followed

310 closely by MRI, based on continuous decrease in signal intensity on PDw, and validated by his-

311 topathology (Figure 5). These data suggest that remyelination period typically takes 4-9 weeks. 
M\#1 - Remyelinated lesion

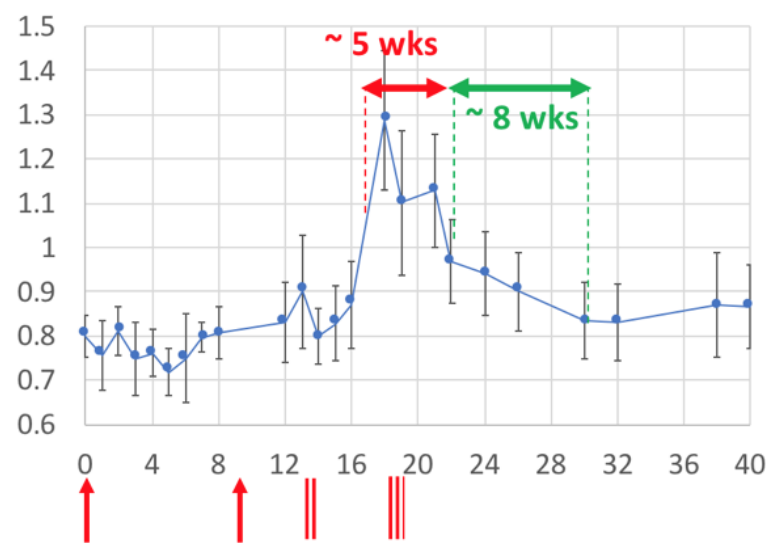

M\#2 - Remyelinated lesion

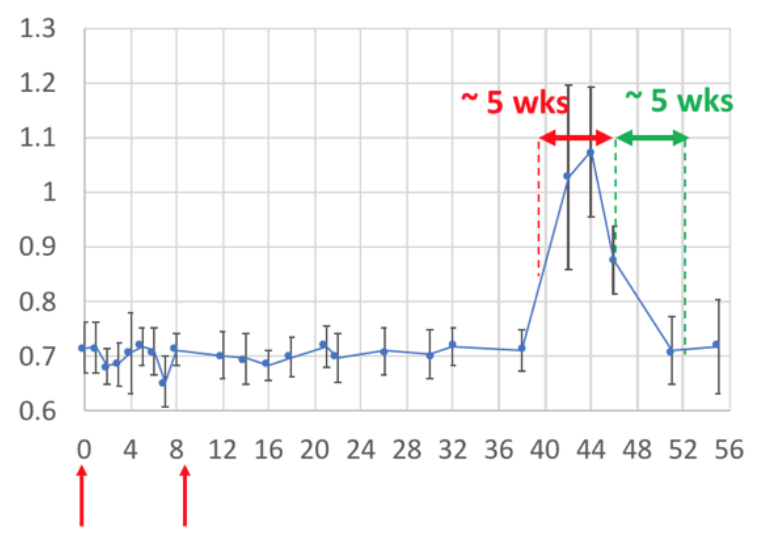

M\#5 - Remyelinated lesion

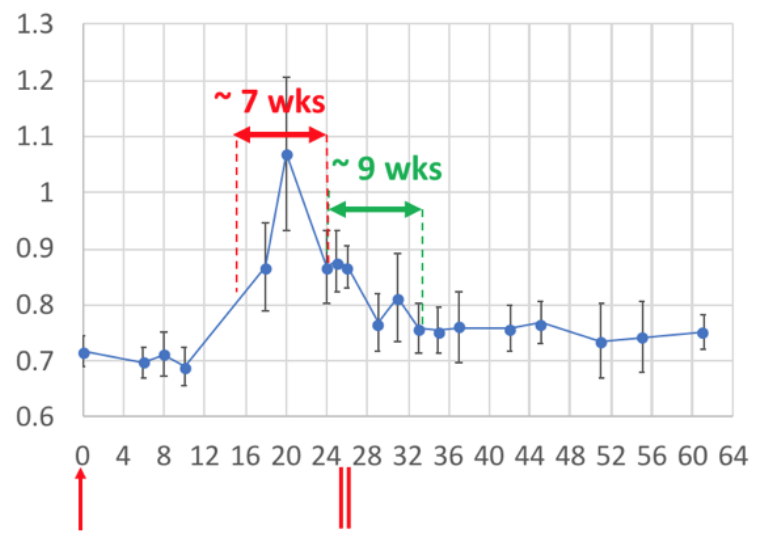

M\#5 - Remyelinated lesion

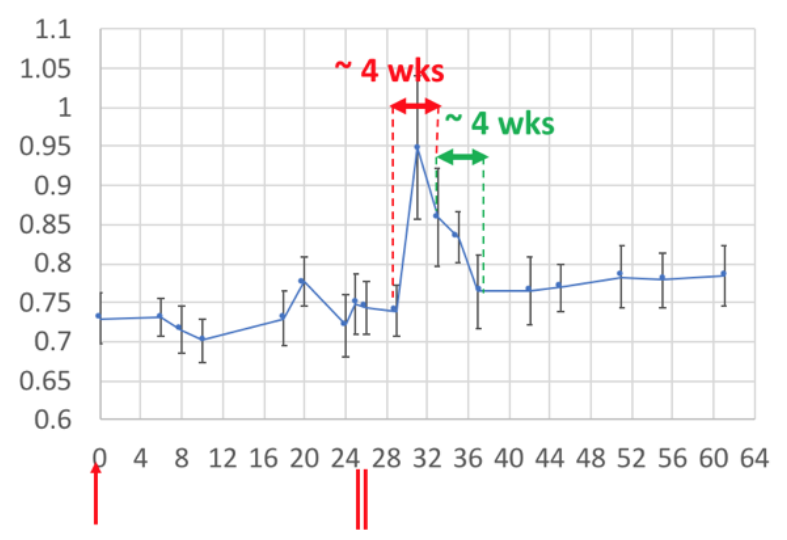

Figure 5: Evolution of in vivo PDw MRI signal intensity of remyelinating EAE lesions shows the typical 4-9-week time course of remyelination. Vertical axis: mean PDw signal intensity relative to gray matter. Horizontal axis: weeks post-immunization. Blue line corresponds to mean PDw signal (with standard deviation) of the segmented lesion as visible on MRI and quantified using a region of interest drawn manually and located in the normal appearing white matter area before the lesion appeared. Normal white matter displays an average normalized signal intensity of $0.65-0.75$. Vertical red arrows indicate EAE immunization. Vertical red bars indicate corticosteroid treatment. Horizontal red double arrows indicate the estimated period of demyelination. Horizontal green double arrows indicate the estimated period of remyelination based on the downward slope of intensity measurement. Green titles indicate that the lesion subtype was confirmed with histopathology counterpart. M\# corresponds to animal number in Table 1. 
329 treated with steroids had no difference in the prevalence of remyelinated lesions. Based on serial

330 PDw MRI, 11 of 22 lesions (50\%) were remyelinated in corticosteroid-treated marmosets,

331 whereas in untreated marmosets, 11 of 21 lesions (52\%) were remyelinated. Based on histo-

332 pathological analysis, 6 of 15 lesions (40\%) in corticosteroid-treated marmosets were remyelin-

333 ated, and 11 of $21(52 \%)$ lesions in untreated marmosets were remyelinated. The point biserial

334 correlation model analysis showed that steroid administration had no significant correlation with

335 remyelination $(p=0.8)$. The average experiment duration also did not differ significantly (40

336 weeks in treated and 43 weeks in untreated marmosets).

338 Remyelination may be sex-dependent

339 Using the point biserial correlation model analysis, there was significant correlation $\left(r^{2}=0.94, p\right.$ $340=0.013)$ favoring males $(n=4)$ over females $(n=2)$ for prevalence of remyelinated lesions. 


\section{DISCUSSION}

342 In this study, we investigated the remyelination process in focal brain lesions of marmoset EAE, a relatively faithful MS model, using high-resolution serial in vivo MRI and histopathology. Our main results show that lesion remyelination is common phenomenon in this model. Indeed, 5 of the 6 marmosets displayed evidence of remyelination on either MRI or histopathology, and nearly half of the 43 lesions identified on MRI showed signs of remyelination. In humans, the prevalence of remyelination is thought to be variable across MS patients and phenotypes, though it is not well estimated $(4,5,23)$. Interestingly, the two animals that did not show remyelination according to in vivo MRI, M\#5 and M\#6, had the shortest disease course (16-18 weeks) compared

to the other 4 animals (40-61 weeks), suggesting that these animals may have had a more aggressive course of the disease - although a single remyelinated lesion was detected histopathologically in M\#5.

Our characterization of remyelinated lesions indicated diminished inflammation, repopulated myelin sheaths in different morphology compared to normal-appearing white matter, and repopulation of both mature oligodendrocytes and OPC. Remyelination in MS lesions also directly correlates with the presence of intralesional oligodendrocytes (4, 32, 33). Assessment of acute demyelinating and chronic demyelinated lesions showed fewer oligodendrocytes, and in this histopathological setting, the lesions were hyperintense on in vivo PDw MRI. Taken together, these data suggest that lesions that initially present as hyperintense foci on PDw MRI and subsequently return to isointensity are in fact remyelinated. In this way, signal intensity time courses can be used to infer lesion myelination status in the setting of early lesion development and recovery, even though PDw MRI itself is not pathologically specific. 
The time course of marmoset EAE lesion development and repair is relatively stereotyped, as demyelination lasts around 6 weeks $(14,24)$, consistent with data from this study (4-7 weeks; lesion signal intensity and volume, without much delay. This finding is in parallel with previous opment, but rarer after years of disease duration in chronic lesions (5). specificity, especially on PDw MRI, corroborates and expands our previous finding that PDw MRI is sensitive to myelin status changes $(24,34)$. Those prior studies primarily focused on demyelination but also included limited data on remyelination, with electron microscopy showing

377 thinned myelin sheaths in remyelinated axons (14). Here, we added comparison of PDw and 378 MTR, since MTR has been used to detect remyelination in vivo $(15,16,35)$, finding that PDw 379 was more sensitive and specific for identifying remyelination. Several groups have reported that MTR may be low in remyelinated lesions compared to normal-appearing white matter because of the presence of incomplete or morphologically different myelin sheaths in previously demyelinated regions $(3,35)$. Unlike MTR, which suffers from signal-to-noise reductions due to its calcu383 lation as a voxel-wise division of random variables, PDw signal intensity is directly measured by the MRI system, and thus PDw may prove more useful for simply discriminating the presence or absence of remyelination, and for characterizing its time course (as was done here). 
To determine whether corticosteroid treatment alters the course of lesion repair, half of the marmosets (for each twin pair, the one that showed lesions first) received 5-day treatment courses.

Our data show that the difference in the prevalence of remyelinated lesions, and the duration of the event, did not differ by treatment group. One possible explanation for this result is that the early initiation of corticosteroid treatment (as soon as the first lesion was detected), resulting in treatment completion before lesions were even 1 week old, might have been premature: the initial demyelination period typically lasts 4-7 weeks before remyelination ensues. It is also possible that reduction of inflammation via corticosteroid treatment could have interfered with the remyelination process by slowing clearance of myelin debris, which is a prerequisite for OPC recruitment (36) and differentiation $(37,38)$, thereby negating any potential direct benefit of corticosteroids on remyelination.

Sex effect on remyelination was also investigated, with results suggesting that males had more remyelinating lesions than females. However, this result must be viewed with caution, since the two female animals (M\#5 and M\#6), twin pair, had shorter and more aggressive disease course compared to the other animals studied here. Furthermore, the lesions on these two animals were mainly $\leq 6$ weeks old, possibly too little for substantial repair to have occurred. would strengthen the study, as nonhuman primates are a scarce resource, we were able to max-

407 imize their use by focusing on individual lesions, rather than on number of animals developed remyelination. Another limitation is that different EAE immunization protocols were applied among marmosets. However, we did not observe any difference in lesion outcome across the 
410 different immunization schemes. Notably, previous studies from our group with a similar variety

411 of EAE induction methods have not shown notable differences in disease course or lesion patho-

412 biology $(14,24)$.

413

414 In conclusion, our study clearly demonstrates in vivo remyelination in the marmoset EAE model,

415 further highlighting the utility of this animal model in investigating the repair of inflammatory

416 demyelination. Remyelination was reliably detected and discriminated using serial in vivo MRI

417 with automated segmentation. Our data indicate that the current model is an excellent avenue for

418 investigating mechanisms of remyelination, and monitoring remyelination in a manner that is

419 easily translatable to clinical studies in human beings. 


\section{ACKNOWLEDGMENT AND COMPETING INTERESTS}

423 This study was funded by the National Institute of Neurological Disorders and Stroke Intramural

424 Research Program and Vertex Pharmaceuticals. We thank Dr. Tianxia Wu for statistical anal-

425 yses. We thank everyone who took part in taking care of the animals and helped with acquisition

426 of MRI scans.

427 


\section{REFERENCES}

429 1. Reich DS, Lucchinetti CF, and Calabresi PA. Multiple Sclerosis. N Engl J Med. 2018;378(2):16980.

2. Lubetzki C, Zalc B, Williams A, Stadelmann C, and Stankoff B. Remyelination in multiple sclerosis: from basic science to clinical translation. Lancet Neurol. 2020;19(8):678-88.

3. Brown RA, Narayanan S, and Arnold DL. Imaging of repeated episodes of demyelination and remyelination in multiple sclerosis. Neuroimage Clin. 2014;6:20-5.

4. Chari DM. Remyelination in multiple sclerosis. Int Rev Neurobiol. 2007;79:589-620.

5. Lassmann H, Brück W, Lucchinetti C, and Rodriguez M. Remyelination in multiple sclerosis. Mult Scler. 1997;3(2):133-6.

6. Kremer D, Göttle P, Flores-Rivera J, Hartung HP, and Küry P. Remyelination in multiple sclerosis: from concept to clinical trials. Curr Opin Neurol. 2019;32(3):378-84.

7. Kipp M, van der Star B, Vogel DY, Puentes F, van der Valk P, Baker D, et al. Experimental in vivo and in vitro models of multiple sclerosis: EAE and beyond. Mult Scler Relat Disord. 2012;1(1):15-28.

8. Kipp M, Nyamoya S, Hochstrasser T, and Amor S. Multiple sclerosis animal models: a clinical and histopathological perspective. Brain Pathol. 2017;27(2):123-37.

9. Jagessar SA, Dijkman K, Dunham J, 't Hart BA, and Kap YS. Experimental Autoimmune Encephalomyelitis in Marmosets. Methods Mol Biol. 2016;1304:171-86.

10. Kap YS, Laman JD, and 't Hart BA. Experimental autoimmune encephalomyelitis in the common marmoset, a bridge between rodent EAE and multiple sclerosis for immunotherapy development. J Neuroimmune Pharmacol. 2010;5(2):220-30.

11. Kap YS, Jagessar SA, Dunham J, and 't Hart BA. The common marmoset as an indispensable animal model for immunotherapy development in multiple sclerosis. Drug Discov Today. 2016;21(8):1200-5.

12. Sati P, Silva AC, van Gelderen P, Gaitan MI, Wohler JE, Jacobson S, et al. In vivo quantification of $\mathrm{T}_{2}$ anisotropy in white matter fibers in marmoset monkeys. Neuroimage. 2012;59(2):979-85.

13. Maggi P, Sati P, and Massacesi L. Magnetic resonance imaging of experimental autoimmune encephalomyelitis in the common marmoset. J Neuroimmunol. 2017;304:86-92.

14. Lee NJ, Ha SK, Sati P, Absinta M, Nair G, Luciano NJ, et al. Potential role of iron in repair of inflammatory demyelinating lesions. J Clin Invest. 2019;129(10):4365-76.

15. Chen JT, Collins DL, Atkins HL, Freedman MS, Arnold DL, and Group CMBS. Magnetization transfer ratio evolution with demyelination and remyelination in multiple sclerosis lesions. Ann Neurol. 2008;63(2):254-62.

16. Chen JT, Easley K, Schneider C, Nakamura K, Kidd GJ, Chang A, et al. Clinically feasible MTR is sensitive to cortical demyelination in MS. Neurology. 2013;80(3):246-52.

17. Filippi M, Rocca MA, Barkhof F, Brück W, Chen JT, Comi G, et al. Association between pathological and MRI findings in multiple sclerosis. Lancet Neurol. 2012;11(4):349-60.

18. Absinta M, Sati P, and Reich DS. Advanced MRI and staging of multiple sclerosis lesions. Nat Rev Neurol. 2016;12(6):358-68.

19. Laule C, and Moore GRW. Myelin water imaging to detect demyelination and remyelination and its validation in pathology. Brain Pathol. 2018;28(5):750-64.

20. Yano R, Hata J, Abe Y, Seki F, Yoshida K, Komaki Y, et al. Quantitative temporal changes in DTI values coupled with histological properties in cuprizone-induced demyelination and remyelination. Neurochem Int. 2018;119:151-8.

21. Schmierer K, Parkes HG, So PW, An SF, Brandner S, Ordidge RJ, et al. High field (9.4 Tesla) magnetic resonance imaging of cortical grey matter lesions in multiple sclerosis. Brain. 2010;133(Pt 3):858-67.

22. Filippi M, Brück W, Chard D, Fazekas F, Geurts JJG, Enzinger C, et al. Association between pathological and MRI findings in multiple sclerosis. Lancet Neurol. 2019;18(2):198-210. 
23. Patrikios P, Stadelmann C, Kutzelnigg A, Rauschka H, Schmidbauer M, Laursen H, et al. Remyelination is extensive in a subset of multiple sclerosis patients. Brain. 2006;129(Pt 12):3165-72.

24. Lee NJ, Ha SK, Sati P, Absinta M, Luciano NJ, Lefeuvre JA, et al. Spatiotemporal distribution of fibrinogen in marmoset and human inflammatory demyelination. Brain. 2018;141(6):1637-49.

25. 't Hart BA, and Massacesi L. Clinical, pathological, and immunologic aspects of the multiple sclerosis model in common marmosets (Callithrix jacchus). J Neuropathol Exp Neurol. 2009;68(4):341-55.

26. Goodin DS. Glucocorticoid treatment of multiple sclerosis. Handb Clin Neurol. 2014;122:45564.

27. Sellebjerg F, Barnes D, Filippini G, Midgard R, Montalban X, Rieckmann P, et al. EFNS guideline on treatment of multiple sclerosis relapses: report of an EFNS task force on treatment of multiple sclerosis relapses. Eur J Neurol. 2005;12(12):939-46.

28. Reich DS. Imag(in)ing multiple sclerosis: Time to take better pictures. J Neuroimmunol. 2017;304:72-80.

29. Roy S, Butman JA, Reich DS, Calaabresi PA, Pham DL. Multiple sclerosis lesion segmentation from brain MRI via fully convolutional neural networks. arXiv Computer Science. arXiv:1803.09172v1; 2018.

30. Roy S, Knutsen A, Korotcov A, Bosomtwi A, Dardzinski B, Butman JA, Pham DL. A deep learning framework for brain extraction in humans and animals with traumatic brain injury. IEEE 15th International Symposium on Biomedical Imaging; 2018.

31. Maggi P, Macri SM, Gaitán MI, Leibovitch E, Wholer JE, Knight HL, et al. The formation of inflammatory demyelinated lesions in cerebral white matter. Ann Neurol. 2014;76(4):594-608.

32. Lucchinetti C, Brück W, Parisi J, Scheithauer B, Rodriguez M, and Lassmann H. Heterogeneity of multiple sclerosis lesions: implications for the pathogenesis of demyelination. Ann Neurol. 2000;47(6):707-17.

33. Lucchinetti C, Brück W, Parisi J, Scheithauer B, Rodriguez M, and Lassmann H. A quantitative analysis of oligodendrocytes in multiple sclerosis lesions. A study of 113 cases. Brain. 1999;122 ( Pt 12):2279-95.

34. Reich DS, White R, Cortese IC, Vuolo L, Shea CD, Collins TL, et al. Sample-size calculations for short-term proof-of-concept studies of tissue protection and repair in multiple sclerosis lesions via conventional clinical imaging. Mult Scler. 2015;21(13):1693-704.

35. Mallik S, Samson RS, Wheeler-Kingshott CA, and Miller DH. Imaging outcomes for trials of remyelination in multiple sclerosis. J Neurol Neurosurg Psychiatry. 2014;85(12):1396-404.

36. Cunha MI, Su M, Cantuti-Castelvetri L, Müller SA, Schifferer M, Djannatian M, et al. Proinflammatory activation following demyelination is required for myelin clearance and oligodendrogenesis. J Exp Med. 2020;217(5).

37. Gruchot J, Weyers V, Göttle P, Förster M, Hartung HP, Küry P, et al. The Molecular Basis for Remyelination Failure in Multiple Sclerosis. Cells. 2019;8(8).

38. Rawji KS, Mishra MK, and Yong VW. Regenerative Capacity of Macrophages for Remyelination. Front Cell Dev Biol. 2016;4:47. 


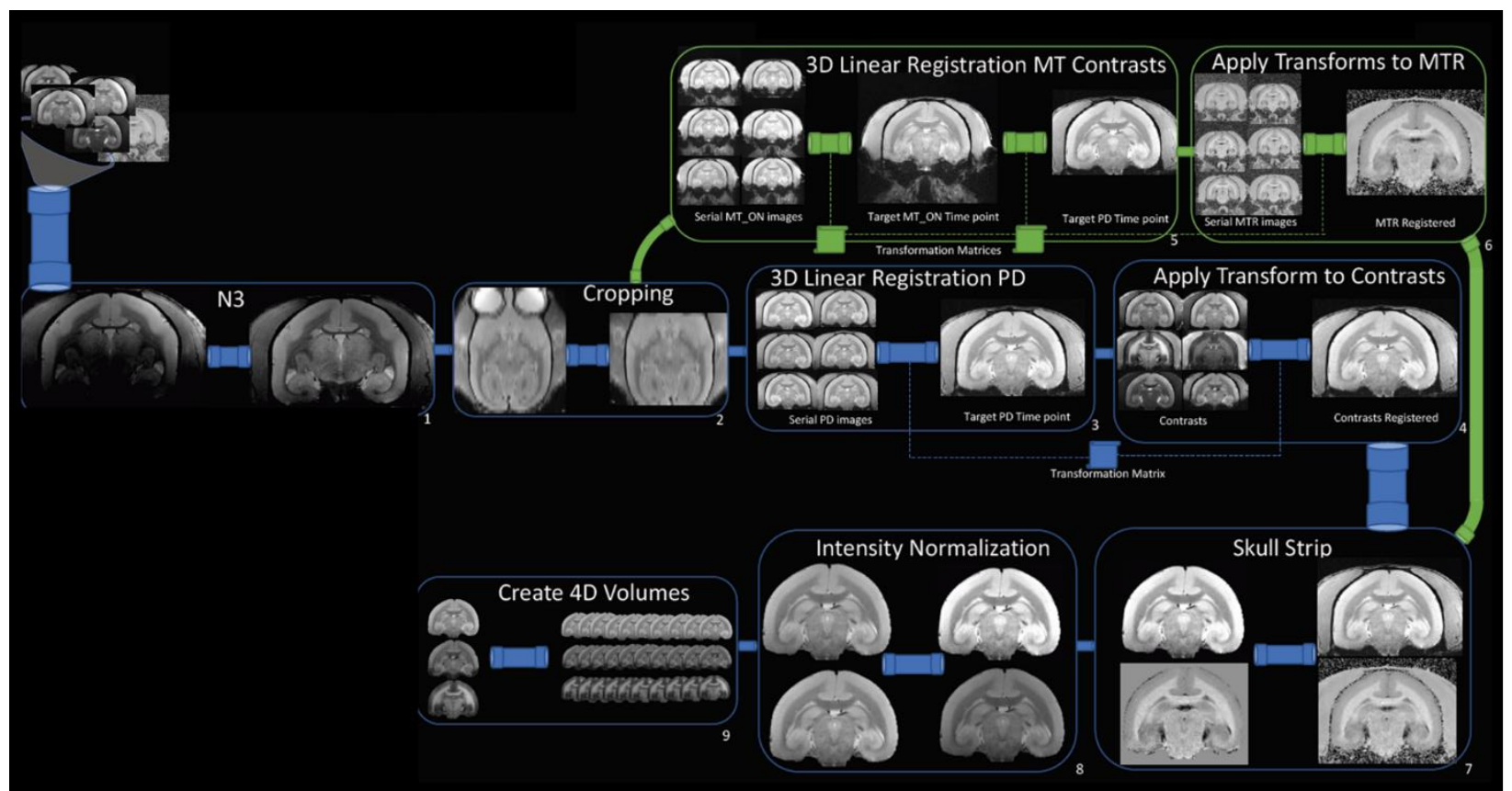

522 Supplementary Figure 1: Workflow of the MRI post-processing pipeline. $\mathrm{PD}=$ proton density; $\mathrm{MT}=$ 523 magnetization transfer; MTR = magnetization transfer ratio; N3 = bias field correction. 


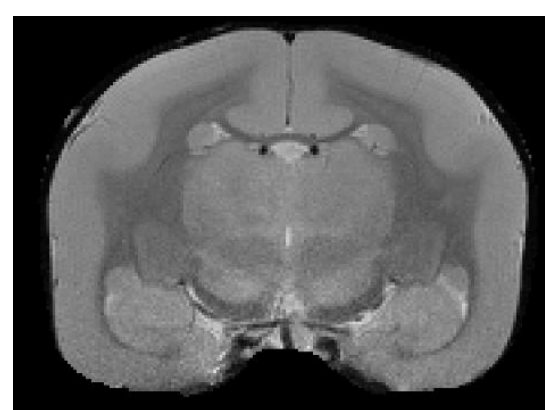

Baseline MRI

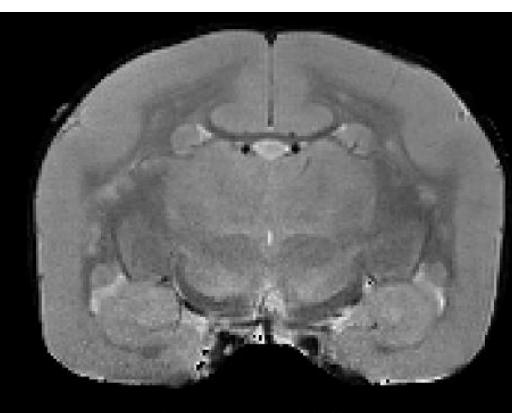

Follow-up MRI

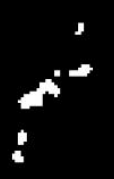

Automated Lesion Segmentation

527 Supplementary Figure 2: Post-processed proton-density weighted images used as input for the convolutional neural network -based lesion segmentation. All hyperintense lesions that appeared on the follow-up MRI performed post-immunization (M\#1, 22 weeks post-immunization) were captured by the automated algorithm. 


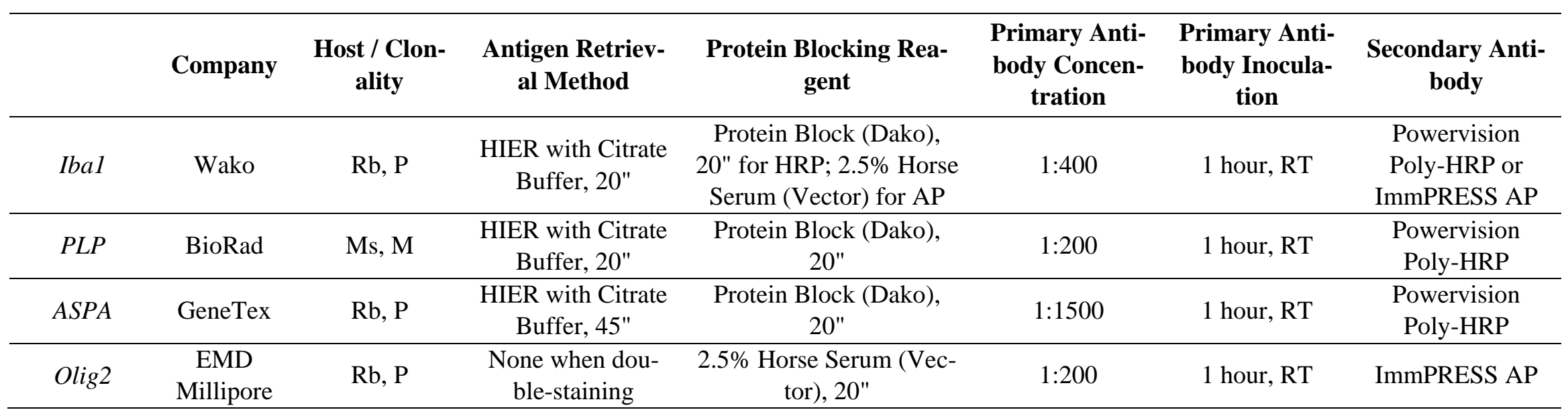

Supplementary Table 1. Immunohistochemistry methodology. For each of the immunohistochemical targets, respective companies, clonalities and hosts, and methods for antigen retrieval, blocking, and primary and secondary antibody inoculation are listed. HIER = heat-induced epitope retrieval; $\mathrm{RT}=$ room temperature; $\mathrm{P}=$ polyclonal antibody; $\mathrm{M}=$ monoclonal antibody; $\mathrm{Rb}=$ rabbit; $\mathrm{Ms}=\mathrm{mouse}$ 
bioRxiv preprint doi: https://doi.org/10.1101/2021.10.27.466044; this version posted October 28, 2021. The copyright holder for this preprint (which was not certified by peer review) is the author/funder, who has granted bioRxiv a license to display the preprint in perpetuity. It is made 537 available under aCC-BY 4.0 International license. 